\title{
Special Issue: Laser-Induced Incandescence
}

\author{
G. Zizak
}

(C) Springer-Verlag 2011

Detection of nanoparticles is an area of particular interest for the scientific community. Nanoparticles are ubiquitous, i.e. carbonaceous nanoparticles, or soot, originating from a variety of anthropogenic combustion sources and biomass burning; they greatly reduce the air quality and affect the public health. On the other side innovative materials are obtained in new industrial processes based upon flame synthesis of nanoparticles with highly specific functionalities. Several techniques are commonly used for the measurement and characterization of nanoparticles.

Laser-Induced Incandescence (LII) is a diagnostic technique that offers numerous advantages. It is an optical, nonintrusive technique that gives a measure of both the concentration and the size of nanoparticles, with the possibility of 2D imaging, thus allowing for the study of the nanoparticle formation processes. Moreover, it is highly sensitive, which is relevant for environmental applications.

The LII technique basically involves a fast heating of the nanoparticles by absorption of laser light on a nanosecond scale and a time-resolved detection of the induced incandescence signal. Depending on the experimental conditions many physical processes are involved that are not completely understood. This was well illustrated in the first workshop on LII held in Duisburg, Germany in 2005 and in the Special issue on Laser-Induced Incandescence of Applied Physics B in 2006. Scientists interested in the develop- ment of the LII technique and in its applications decided to continue the workshop series to share their studies on best practices, modeling and experimental data evaluation. After the workshops of Bad Herrenalb, Germany, in 2006 and Ottawa, Canada, in 2008 a Fourth International Workshop on Laser-Induced Incandescence: Quantitative Interpretation, Modeling Applications was held in Varenna, Italy, in April 2010. An increasing number of registered participants and scientific contributions is observed through the workshops, indicating a growing interest in the LII technique by researchers of different scientific areas and from all over the world.

This special issue of Applied Physics B contains 15 selected peer-reviewed papers related to the contributions presented in the Varenna workshop. The themes regard the determination of optical properties of the soot and the influence on LII signals, the modeling and validation of LII signals, the integration of LII with other experimental techniques and the use of LII in several challenging experimental situations, such as engines, turbulent flames, high pressure or vacuum conditions.

We want to express our gratitude to Editor-in-Chief Frank Träger and Jutta Kaisig of the University of Kassel for their advice and support in preparing this special issue.

Milan, April 2011
G. Zizak ( $\varangle)$

IENI, Consiglio Nazionale delle Ricerche, via Cozzi 53, 20125

Milan, Italy

e-mail: zizak@ieni.cnr.it

Fax: +39-02-66173321 